\title{
Lessons from a decade of individual-based models for infectious disease transmission: a systematic review (2006-2015)
}

\author{
Lander Willem $^{1 *}$ (D), Frederik Verelst ${ }^{1}$, Joke Bilcke ${ }^{1}$, Niel Hens ${ }^{1,2}$ and Philippe Beutels ${ }^{1,3}$
}

\begin{abstract}
Background: Individual-based models (IBMs) are useful to simulate events subject to stochasticity and/or heterogeneity, and have become well established to model the potential (re)emergence of pathogens (e.g., pandemic influenza, bioterrorism). Individual heterogeneity at the host and pathogen level is increasingly documented to influence transmission of endemic diseases and it is well understood that the final stages of elimination strategies for vaccine-preventable childhood diseases (e.g., polio, measles) are subject to stochasticity. Even so it appears IBMs for both these phenomena are not well established. We review a decade of IBM publications aiming to obtain insights in their advantages, pitfalls and rationale for use and to make recommendations facilitating knowledge transfer within and across disciplines.
\end{abstract}

Methods: We systematically identified publications in Web of Science and PubMed from 2006-2015 based on title/abstract/keywords screening (and full-text if necessary) to retrieve topics, modeling purposes and general specifications. We extracted detailed modeling features from papers on established vaccine-preventable childhood diseases based on full-text screening.

Results: We identified 698 papers, which applied an IBM for infectious disease transmission, and listed these in a reference database, describing their general characteristics. The diversity of disease-topics and overall publication frequency have increased over time (38 to 115 annual publications from 2006 to 2015). The inclusion of intervention strategies (8 to 52) and economic consequences ( 1 to 20) are increasing, to the detriment of purely theoretical explorations. Unfortunately, terminology used to describe IBMs is inconsistent and ambiguous. We retrieved 24 studies on a vaccine-preventable childhood disease (covering 7 different diseases), with publication frequency increasing from the first such study published in 2008. IBMs have been useful to explore heterogeneous betweenand within-host interactions, but combined applications are still sparse. The amount of missing information on model characteristics and study design is remarkable.

Conclusions: IBMs are suited to combine heterogeneous within- and between-host interactions, which offers many opportunities, especially to analyze targeted interventions for endemic infections. We advocate the exchange of (open-source) platforms and stress the need for consistent "branding". Using (existing) conventions and reporting protocols would stimulate cross-fertilization between research groups and fields, and ultimately policy making in decades to come.

Keywords: Individual-based, Agent-based, Mathematical epidemiology, Modeling, Emerging diseases, Endemic diseases, Transmission, Dynamics, Networks, ODD protocol

\footnotetext{
*Correspondence: lander.willem@uantwerp.be

${ }^{1}$ Centre for Health Economics Research \& Modeling Infectious Diseases,

Vaccine and Infectious Disease Institute, University of Antwerp, Antwerp,

Belgium

Full list of author information is available at the end of the article
} 


\section{Background}

Infectious diseases have substantial impact on public health, health care, macroeconomics and society. The availability of options to control and prevent the emergence, expansion or resurgence of pathogens warrants continuous evaluation using different methods. Mathematical models provide a powerful set of tools in this process, as timely, budgetary or ethically feasible alternatives are often lacking (e.g., school closure interventions or vaccine trials to study herd immunity effects) [1]. Even in countries or regions with high overall levels of vaccination coverage and herd immunity, sporadic outbreaks may still occur. For instance, in Europe, the flow of refugees through countries with ongoing large measles outbreaks (e.g., Bosnia-Herzegovina, Serbia) increased the risk of stochastic introduction events elsewhere [2]. Model-based evaluations can be useful to understand the behavioral mechanisms influencing the frequency, peak and duration of these outbreaks, with the aim to design (better) strategies to prevent them or minimize their impact [3].

Transmission dynamics of infectious diseases are usually modeled at the population-level with a compartmental model, and less frequently till now at the individual level with an individual-based model (IBM). A compartmental model tracks changes in compartments without specifying which individuals are involved [4]. Compartmentalization typically reflects health states relevant for transmission (e.g., susceptible, infectious and recovered), though more partitioning is possible according to age and/or other relevant host characteristics. Heterogeneous and temporal behavior is modeled through incorporation of relevant time-dependent social mixing, community structures and seasonality, relevant for infectious disease dynamics [5-7]. Process dynamics are captured in transition rates, representing the rate by which an average individual transitions between compartments. IBMs work bottom-up, with population-level behavior emerging from the interactions between autonomous individuals and their environment [8]. They allow the history of every individual to be tracked and network structures to be explicitly represented [4]. Each individual has a unique set of attributes or state variables that can change through time including spatial location, physiological traits and/or social behavior $[9,10]$. As such, IBMs allow a high degree of heterogeneity for the creation, disappearance and movement of a finite collection of discrete interacting individuals $[8,11]$.

Deterministic models have been very useful to simulate the dynamics of endemic infections, but they are less suited to simulate events that are subject to chance [4]. For instance, the (non-)propagation of an infection in the initial stages of an emerging disease or in the final stages of elimination is dominated by individual heterogeneity and random events. The interplay between infectious disease dynamics and individual human behavior can be key to improve control efforts [12, 13]. Both compartmental and individual-based modeling approaches can simulate stochastic events. A compartmental model design, based on the epidemiological status of the population and known disease aspects, can be used in combination with stochastic and time-varying disease transmission rates [14]. As such, stochastic terms provide model flexibility to accommodate changes in the transmission rate that might occur due to unobserved processes. Remarkable progress has also been made with meta-population models to incorporate heterogeneous and temporal aspects by considering stochastic inter-population mobility [15]. For example, the Global Epidemic and Mobility (GLEaM) model, has been used to assess international travel restrictions during the 2009 influenza pandemic and the 2014-2016 Ebola outbreak [16-19]. Stochastic IBMs allow even more variation due to chance, which is especially of interest to study systems with small susceptible populations due to the context (e.g., a hospital or small island) or due to high population immunity (e.g., by routine childhood immunization programs).

Vaccination is one of the most effective tools to prevent infectious diseases and their consequences [20]. High immunization coverage is extremely important at the community level to protect patients who cannot be vaccinated due to medical reasons or age (e.g., the very young or very old). Indeed, also older age groups benefit from childhood immunization, for example the administration of conjugate pneumococcal vaccine to young children has had a substantial impact on adult pneumococcal disease [21]. However, low incidence of vaccinepreventable diseases in many high- and middle-income countries, often leads to the public perception of reduced severity and susceptibility [20]. Combined with rising concerns about real or perceived adverse events, the apparent absence of disease leads people to delay or refuse vaccinations more often [22]. Outbreaks of vaccinepreventable disease in countries with historically successful vaccination programs can take off in immigrant or unvaccinated pockets of susceptibles and potentially affect vulnerable groups such as infants and the immunocompromised [23]. Modeling the stochastic nature of transmission events in highly immunized populations with (clustered) heterogeneity in susceptibility can benefit from an IBM approach. To investigate the frequency and methods of such IBM applications, we focus on vaccine-preventable childhood diseases in a subsection of this review.

Different terminology has been used for individuallevel models including agent-based model (ABM), cellular automata (CA), micro-simulation as well as more generic 
terms such as computer simulations and complex adaptive systems. A distinction in nomenclature can be designated by whether the simulation is based on nodes of a grid (as in a CA), or based on agents that are self-contained programs that collect information from their surroundings and have the autonomy and capacity to learn and adapt (ABM) [24]. These terms have been used interchangeably in the literature $[25,26]$. Henceforth, we will use the overall term "IBM" to refer to the individual-level approach.

Describing the methodology of an IBM is more difficult compared to compartmental models, which often can be formulated in the general language of mathematics [27]. Published IBM methodology is often incomplete or ambiguous and therefore less accessible or reproducible [28]. In 2006, a board of 28 modelers developed and tested a generic format to document IBM research consisting of three blocks: Overview, Design concepts, and Details (ODD) [28]. The primary objective was to make model descriptions more understandable and complete. The "Overview" should provide readers the modeling focus, resolution and complexity based on the declaration of the model entities and the scheduling of the processes. The "Design Concept" describes the general approach to establish a link with emergence, the type of interactions and if/how stochasticity is considered. The "Details" section should contain all information required to completely reimplement the model and run the baseline simulations. In 2010, the ODD protocol has been revised and was used in at least 50 publications though still many papers lacked a standard approach to describe the IBM [29].

In this systematic review, we summarize and discuss IBM applications and terminology across different epidemiological disciplines, published between 2006 and 2015. We elaborate in general on the different modeling topics and purposes over time and identify research and data gaps. As indicated above we also focus on IBM research for childhood diseases with a long history of vaccination, i.e. on risk assessment and elimination strategies in heterogeneous settings with high populationimmunity. We extract and discuss model characteristics such as the implementation of social mixing, demographic evolution over time, as well as the modeling platforms for IBMs. For these applications, we aimed to identify the rationale for an IBM and provide model characteristics and recommendations to enhance knowledge transfer across disciplines.

\section{Methods}

Our search, extracting and reporting strategy is based on the evidence-based protocol PRISMA (Preferred Reporting Items for Systematic Reviews and Meta-Analyses) [30] and the Cochrane guidelines [31]. We use IBM as the overarching term for models at the individual-level, also noted as ABM, CA, micro-simulation, etc. We conducted a systematic review of studies using an IBM for infectious disease transmission, using this definitions based on the literature:

Infectious diseases: "Caused by pathogenic microorganisms, such as bacteria, viruses, parasites or fungi; the diseases can be spread, directly or indirectly, from one person to another. Zoonotic diseases are infectious diseases of animals that can cause disease when transmitted to humans" [32].

Individual-based model: "Computer simulation for the creation, disappearance and movement of a finite collection of interacting individuals or agents with unique attributes regarding spatial location, physiological traits and/or social behavior" [8-11, 25, 33].

\section{Search}

We searched PubMed and Web of Science Core Collection using Endnote (X7.2.1) for English language articles published from January 2006 up to December 2015. Based on the listed definitions and exploratory searches, the following search query was used on January 3, 2016: "(model* OR simulat*) AND (agent-based OR individual-based $O R$ individual-level OR multi-agent $O R$ actor-based $O R$ micro-simulation OR microsimulation OR cel" automata OR (stochastic AND individual*)) AND (disease OR infect* OR transmi* OR epidem*)". Pubmed and Web of Science both ignore hyphens in the search query, so e.g., "individual based" and "agent based" were also retrieved. In line with Cochrane guidelines, eligibility criteria were agreed upon by four researchers (LW, JB, NH and PB, experienced in infectious disease and/or individual-based modeling) prior to screening. We included original research papers using an IBM with a focus on infectious disease transmission in humans. I.e., reviews and studies related to animal research, ecology, molecular biology and immunology were excluded. The screening on title/abstract/keywords and full-text if necessary was conducted by a single reviewer (LW), in consultation with co-authors in case of doubt.

\section{Model classification}

For each study that met the eligibility criteria, LW and FV retrieved independently the topic (disease), the modeling purpose (methods, dynamics or interventions) and model specifications such as setting, economic analysis, reference data, open-source initiatives and sustainability based on model names. We classified the modeling purpose according to the following definitions: (1) methods: describing new approaches for IBM 
research by introducing modeling concepts, performance enhancements or emulation techniques; (2) dynamics: using a methodology to understand transmission dynamics and elaborate on the effect of model assumptions and parameter values on the results; (3) interventions: to evaluate intervention measures to inform policy makers, using a methodology and based on knowledge on the transmission dynamics. Studies for which LW and FV disagreed with respect to classification were discussed up to when agreement was reached.

\section{Full-text screening}

To extract model characteristics and applications, a fulltext screening was done in duplicate by LW and FV for papers on vaccine-preventable childhood diseases, defined as the diseases included in the immunization recommendations between birth and 15 months by the Centers for Disease Control and Prevention [34]. As such, we included diphtheria, Haemophilus influenzae type b, hepatitis A, hepatitis $B$, influenza, measles, meningococcus, mumps, pertussis, pneumococcus, polio, rotavirus, rubella, tetanus and varicella. Papers on influenza were excluded from the full-text analysis to focus on limited stochastic outbreaks in heterogeneous populations with high levels of herd immunity. For more info on forecasting influenza outbreaks, we refer to the systematic review by Nsoesie et al. [35]. For each full-text article, we listed the topic, the setting, model specifications (e.g., state variables, time horizon, step size), design of experiments (e.g., realizations, platform), the added value of an IBM compared to deterministic alternatives and the terminology.

\section{Results}

Using the online databases PubMed and Web of Science, we identified 5520 unique articles published between 2006-2015 matching the search criteria listed in the "Methods" section. Our query included many general descriptions for IBMs and infectious disease transmission to decrease the number of false negative hits. Based on title, abstract and keyword screening with predefined eligibility criteria (see "Methods"), we excluded 4761 articles. More specifically, we excluded over 800 articles on a different topic (stock markets, oncology, engineering, non-human, etc.) and many more with a stochastic model but not at the individual-level. Other infectious disease IBMs did not include transmission events. We analyzed full-texts for 100 abstracts containing an unclear or incomplete model description and excluded 62 of them. Finally, we obtained 698 studies using an IBM to simulate infectious disease transmission. The adapted PRISMA diagram of the screening process with inclusion and exclusion criteria can be found in Additional file 1. In this main text, we describe and discuss general findings and provide the complete set of references with study characteristics as Additional file 2 .

\section{Modeling purpose}

Among the 698 included studies, we observed an absolute increase in the annual number of IBM publications (38 to 115 from 2006 to 2016) and the diversity of disease-topics (Fig. 1). Most papers in our selection are on unspecified close-contact diseases (27\%), closely followed by influenza (23\%). Many studies in the latter group were published shortly after the $2009 \mathrm{H} 1 \mathrm{~N} 1$ pandemic [36-38]. A similar event-related trend is observed for Ebola in 2015 [39, 40] and for bioterrorism subjects, with 13 studies between 2006 and 2013, expressing the rising concerns over smallpox [41], anthrax [42] and pneumonic plague [43]. Table 1 presents an overview of the different topics, modeling purposes and study characteristics. We observed that models for general close-contact diseases are mostly used to describe methodology and transmission dynamics. In contrast, many studies on influenza are conducted to control seasonal or pandemic outbreaks with vaccination programs or social distancing such as isolation and school closures $[44,45]$. In recent years, we observe a shift for the use of IBMs from methodological (43\% to 19\%) to application and intervention-related purposes (21\% to $44 \%)$. This is entangled in the rising number of articles on the transmission and control of human immunodeficiency virus (HIV), human papillomavirus (HPV), malaria, tuberculosis and methicillin-resistant $S$. aureus. Studies on sexually transmitted infections increasingly tend to evaluate screening strategies in the general population, compared to previous studies focusing on prevention measures for men who have sex with men or injecting drug users. We observed an accelerating trend in economic analyses using an IBM from 1 study in 2006 up to 20 in 2015. Malaria is the dominant topic for vector-borne disease models, covering drugs and vector control but also, more recently, potential malaria vaccination options [46-48]. Dengue has also been modeled using IBMs, though usually with the primary aim to understand the transmission dynamics, pathogenicity and epidemiology rather than to inform policy makers [49]. Many other diseases have also occasionally been modeled using IBMs including the respiratory syncytial virus [50] and cholera [51]. IBM studies on vaccine-preventable childhood diseases appeared in 2008 for measles and pneumococcus, accumulating to 24 studies by the end of 2015 covering meningococcus, varicella, polio, pertussis and hepatitis A (see "Full-text analysis" subsection for more details).

Methodological papers, not applied to a specified closecontact infection, mostly describe the conceptual usage of an IBM to simulate heterogeneous disease dynamics and targeted intervention strategies. Other studies were published on validation procedures $[52,53]$, performance 

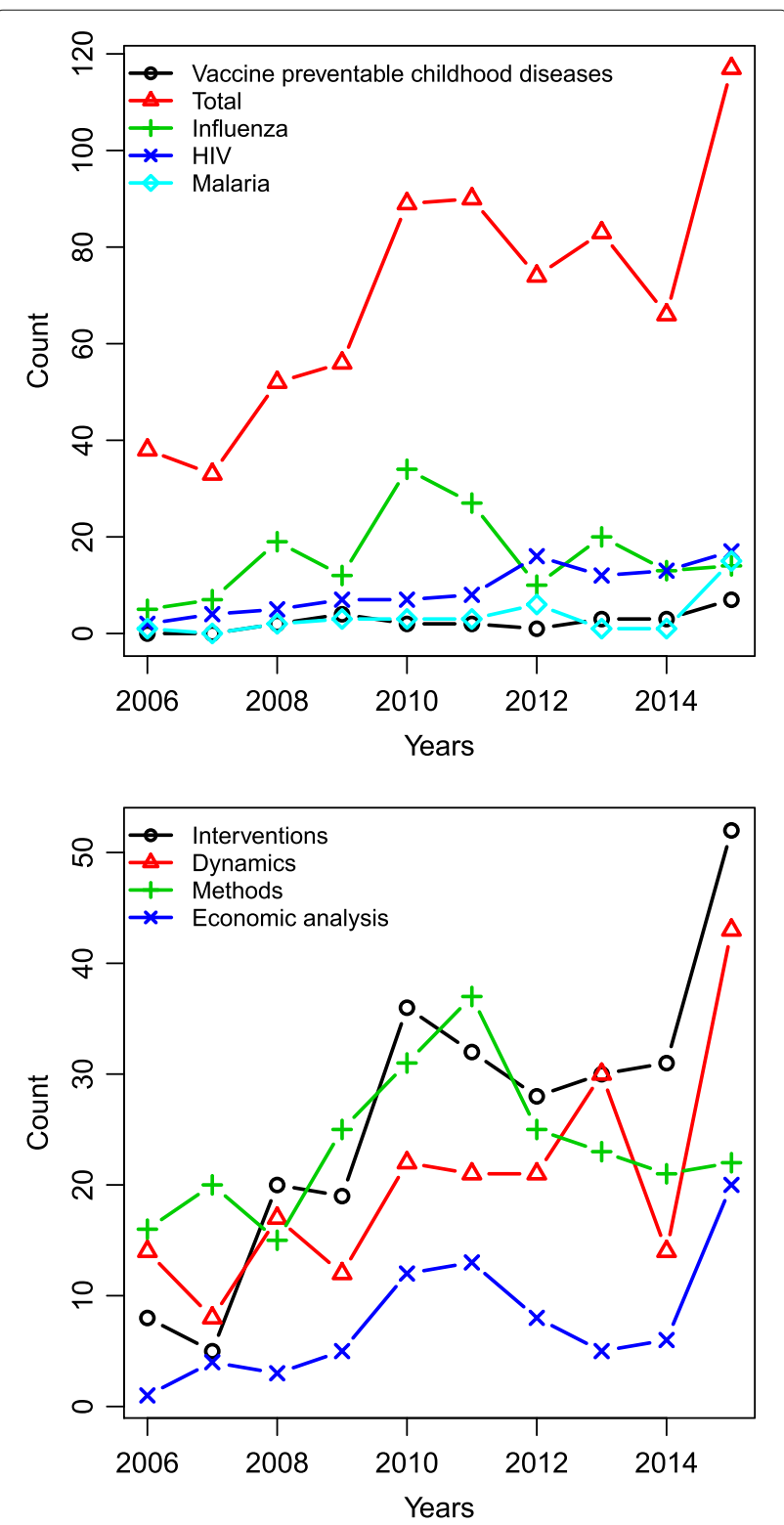

Fig. 1 IBM studies published over time by topic (top) and purpose (bottom)

issues $[54,55]$ and emulation to improve rapid policy making in various settings [55-58]. Models and model output have been calibrated and validated with observed incidence and (sero)prevalence data $[49,59,60]$ but also with data generated by other models, such as deterministic ordinary differential equation models [61] or metapopulations models [62].

As supplementary analysis, we explored the relative number of studies over time that have or have not used an IBM to model infectious disease transmission (described in Additional file 3). We performed additional literature queries considering the number of records in Web of Science as a proxy for the effective number of modeling studies and a constant fraction of false positives and negatives over time. As such, we observed that the yearly number of published IBM related studies tends to increase more rapidly since 2006 compared to the annual publications on modeling infectious disease transmission in general.

\section{Terminology}

We observed a variety of descriptions for models simulating transmission events between humans at the individual-level. Table 2 illustrates the presence of query terms in all unique hits and for the selected subset of IBMs for infectious disease transmission. The positive predictive value represents the proportion of positive results that are truly positive (i.e. the proportion of query records included after screening). We also estimated the sensitivity, namely the probability of detection, as the proportion of positives that are correctly identified as such. Of the 698 included studies, 12 did not contain "model" in their title, abstract or keywords. To describe the individual-level characteristics, ABM and IBM were mainly used, followed by CA or micro-simulation (though with different spelling variations). Other terminology that covered our definitions included "individual-level model" [63], "individually based SIR model" [64], "small world network" [65], "largescale stochastic simulation" [66], "equation free approach" [67] or other variants of "stochastic models" [36, 68]. General keywords gave many false positive hits though still resulted in 124 papers that did not use the most common terminology in their abstract, title or keywords. None of our disease related query terms were used by the complete set of IBM papers on infectious disease transmission and a low positive predicted value was observed. Firstly, the term "disease" is also valid for chronic and lifestyle diseases. Secondly, we needed to include general terms such as "transmi" or "epidem" to capture papers only describing their specific disease topic like influenza [69] or dengue [70]. Unfortunately, "transmi*" caused many false positive hits for research on power markets, sensors and information networks.

\section{Modeling group diversity and branding}

Based on their acronym, some models were identified as having been applied multiple times, for example STDSIM [71], EPISIMS [72], EMOD [73], ONCHOSIM [74], HPV-ADVISE [75], FRED [76] and the Openmalaria platform [77]. This non-exhaustive list covers models for airborne, sexually transmitted, parasitic and vector-borne diseases. With such consistent acronyms, one can link studies for different diseases, such as STDSIM developed for HIV [71] but used for HPV [78] and herpes simplex [79] or FRED implemented for influenza [76] and recently used for measles [80], or EMOD used for HIV [81] and malaria [82]. In addition, we identified studies 
Table 1 Characteristics of IBMs studies for infectious disease transmission published from 2006 to 2015

\begin{tabular}{|c|c|c|c|c|c|c|c|c|c|c|}
\hline \multicolumn{2}{|l|}{ Topic } & \multirow[t]{2}{*}{ Count } & \multicolumn{3}{|c|}{ Purpose } & \multicolumn{4}{|c|}{ Strategy } & \multirow{2}{*}{$\begin{array}{l}\text { Economic } \\
\text { Analysis }\end{array}$} \\
\hline Pathogen & Type & & Methods & Dynamics & Interventions & Vaccine & NPI & Drugs & Screening & \\
\hline Unspecified Close-contact & General & 186 & 95 & 77 & 14 & 5 & 9 & 1 & - & 4 \\
\hline Unspecified STI & General & 9 & 3 & 5 & 1 & 1 & 1 & - & 1 & - \\
\hline Unspecified Vector-borne & General & 7 & 7 & - & - & - & - & - & - & - \\
\hline Bioterrorism & General & 6 & 3 & 1 & 2 & - & 2 & 1 & - & 1 \\
\hline Influenza & Viral & 161 & 51 & 37 & 73 & 37 & 47 & 24 & 3 & 14 \\
\hline HIV & Viral & 91 & 25 & 25 & 41 & 3 & 12 & 25 & 15 & 23 \\
\hline HPV & Viral & 27 & 2 & 2 & 23 & 23 & - & - & 7 & 15 \\
\hline Hepatitis C & Viral & 12 & 2 & 3 & 7 & 1 & - & 6 & 2 & 3 \\
\hline Ebola & Viral & 8 & - & 5 & 3 & 1 & 3 & 2 & - & - \\
\hline SARS & Viral & 8 & 4 & 3 & 1 & 1 & - & - & 1 & - \\
\hline Smallpox & Viral & 7 & - & - & 7 & 7 & 3 & - & 3 & - \\
\hline Measles & Viral & 5 & 1 & 1 & 3 & 3 & - & - & 1 & - \\
\hline Polio & Viral & 4 & 1 & 2 & 1 & 1 & - & - & - & - \\
\hline HIV+Hepatitis C & Viral & 3 & 1 & 1 & 1 & - & 1 & - & - & - \\
\hline HIV+HSV & Viral & 3 & - & 1 & 2 & 1 & - & 1 & - & 1 \\
\hline Varicella Zoster & Viral & 3 & 1 & 2 & - & - & - & - & - & - \\
\hline Respiratory syncytial virus & Viral & 2 & - & - & 2 & 2 & - & - & - & 1 \\
\hline $\begin{array}{l}\text { Acute hemorrhagic } \\
\text { conjunctivitis }\end{array}$ & Viral & 2 & 2 & - & - & - & - & - & - & - \\
\hline Hepatitis A & Viral & 1 & - & - & 1 & 1 & 3 & - & - & - \\
\hline Norovirus & Viral & 1 & - & 1 & - & - & - & - & - & - \\
\hline Malaria & Vector-borne & 35 & 7 & 5 & 23 & 7 & $1-$ & 11 & 3 & 5 \\
\hline Dengue & Vector-borne & 13 & 4 & 5 & 4 & 2 & 3 & - & - & - \\
\hline Chikungunya & Vector-borne & 1 & - & - & 1 & - & 1 & - & - & - \\
\hline Schistosoma & Parasitic & 3 & 2 & 1 & - & - & - & - & - & - \\
\hline Wuchereria & Parasitic & 3 & 2 & - & 1 & - & 1 & 1 & - & - \\
\hline Helminths & Parasitic & 2 & - & 1 & 1 & - & - & 1 & - & - \\
\hline Onchocerca & Parasitic & 2 & - & - & 2 & - & - & 2 & - & 1 \\
\hline Chagas disease & Parasitic & 1 & 1 & - & - & - & - & - & - & - \\
\hline Toxocara & Parasitic & 1 & - & 1 & - & - & - & - & - & - \\
\hline Cryptosporidium & Parasitic & 1 & 1 & - & - & - & - & - & - & - \\
\hline Tuberculosis & Bacterial & 26 & 8 & 6 & 12 & - & - & 9 & 9 & 4 \\
\hline MRSA & Bacterial & 14 & 3 & 2 & 9 & - & 13 & 2 & 4 & 1 \\
\hline Chlamydia & Bacterial & 7 & 3 & - & 4 & 1 & - & - & 3 & 1 \\
\hline Nosocomial infections & Bacterial & 7 & 3 & 2 & 2 & 1 & 4 & - & - & - \\
\hline Syphilis & Bacterial & 6 & - & - & 6 & - & 2 & 1 & 4 & 1 \\
\hline Pneumococcus & Bacterial & 5 & 1 & 1 & 3 & 2 & 1 & - & - & - \\
\hline Cholera & Bacterial & 4 & - & 4 & - & - & - & - & - & - \\
\hline Lepra & Bacterial & 4 & - & 2 & 2 & 1 & - & 2 & 1 & - \\
\hline Gonorrhoea & Bacterial & 3 & - & 1 & 2 & 1 & - & - & 1 & - \\
\hline Clostridium difficile & bacterial & 3 & - & - & 3 & - & 7 & 2 & 2 & - \\
\hline Pertussis & bacterial & 3 & - & 1 & 2 & 2 & - & - & - & 1 \\
\hline Meningococcus & bacterial & 3 & 1 & 1 & 1 & 1 & - & - & - & - \\
\hline Acinetobacter baumannii & bacterial & 1 & - & - & 1 & - & 2 & - & - & - \\
\hline Enterococcus & bacterial & 1 & - & 1 & - & - & - & - & - & - \\
\hline Typhoid & bacterial & 1 & - & 1 & - & - & - & - & - & - \\
\hline Mycobacterium ulcerans & bacterial & 1 & - & 1 & - & - & - & - & - & 1 \\
\hline Foot and mouth disease & zoonose & 1 & 1 & - & - & - & - & - & - & - \\
\hline Total & & 698 & 235 & 202 & 261 & 105 & 125 & 91 & 60 & 77 \\
\hline
\end{tabular}

Each study was assigned one purpose, which is cumulative from methods, dynamics to intervention (e.g., studies about interventions can also describe dynamics and methods). The category "NPI" includes all non-pharmaceutical intervention strategies such as social distancing, school closure and improving standards of living and (hand-)hygiene. HIV: human immunodeficiency virus, HPV: human papillomavirus, HSV: herpes simplex virus, MRSA: methicillin-resistant S. aureus, STI: sexually transmitted infection 
Table 2 Terminology in abstract, title and keywords from all unique query hits and in the included IBM modeling studies for infectious disease transmission. One article can contain several terms

\begin{tabular}{lllll}
\hline & All hits & Included articles & Positive predicted value & Sensitivity \\
\hline Model* $^{*}$ & 5271 & 686 & 0.130 & 0.983 \\
Simulat* & 2318 & 504 & 0.217 & 0.722 \\
Agent-based & 969 & 251 & 0.259 & 0.360 \\
Individual-based & 616 & 241 & 0.391 & 0.345 \\
Micro-simulation & 396 & 54 & 0.136 & 0.249 \\
Cel* automata $^{*}$ & 249 & 62 & 0.037 & 0.077 \\
Other individual-level related terms (see Methods) & 3367 & 124 & 0.159 & 0.178 \\
Disease & 2791 & 445 & 0.285 & 0.638 \\
Infect* & 1939 & 553 & 0.239 & 0.792 \\
Transmi* & 1847 & 441 & 0.172 & 0.632 \\
Epidem* & 3029 & 521 & 0.176 & 0.746 \\
Disease OR infect* & 3564 & 629 & 0.401 & 0.901 \\
Infect* AND disease AND transmi* & 628 & 252 & 0.361 \\
TOTAL & 5520 & 698 & 1.000 \\
\hline
\end{tabular}

The asterisk $\left(^{*}\right)$ is used in the search as a wildcard and represents any group of characters, including no character

that were published by the same authors but links with previous research were not mentioned, at least not in the abstract. Based on authorship, research institute and project names, we could also link other studies to the Openmalaria platform [77] and FRED [76]. Providing IBM code open-source to the research community is not common practice but exists, for example with FluTE [36] and FRED [76].

\section{Full-text analysis}

We analyzed 24 full-text articles on vaccine-preventable childhood diseases, excluding influenza to focus on limited stochastic outbreaks in heterogeneous populations and given the recent systematic review for influenza by Nsoesie et al. [35]. The articles covered transmission dynamics for hepatitis $\mathrm{A}$, measles, meningococcus, pertussis, pneumococcus, polio and varicella. Our search did not yield studies for diptheria, Haemophilus influenzae type $b$, hepatitis B, mumps, rotavirus, rubella or tetanus. In the remainder of this section, we summarize the main findings from the full-text analysis, the per-study details of which can also be found in Table 3.

\section{Purpose}

We retrieved 2 papers that explored methodology to incorporate heterogeneous interactions in a (geo)spatial context [24, 83]. Eight papers elaborated on transmission dynamics, focusing on the influence of social mixing patterns or within-host dynamics [23, 84-90]. Additionally, 14 studies modeled intervention strategies to mitigate infectious disease outbreaks. The majority (12/14) of these studies modeled vaccination campaigns targeting general $[80,91]$ or insufficiently immunized subgroups [92, 93], expanding booster campaigns $[94,95]$, the occurrence of rare adverse events such as vaccine-induced polio [96] and serotype carriage and replacement [97-101]. Two other studies on intervention strategies evaluated social distancing options and adaptive social contact behavior $[102,103]$.

\section{Setting}

We found papers modeling a theoretical grid $[83,85,96]$ or a generic "low income setting" [89]. The study population of the other papers did not exceed a single country, and ranged from a North American $[23,24,80,86,90,92,94,101]$ to a European $[84,87,88,95,97-100,102,103]$ or African $[91,93]$ country.

\section{State variables}

The lowest-level entity in each model was a "person" and the minimum characteristic was the health state. Depending on the research questions, also heterogeneity for age, gender, spatial location, social mixing behavior [103], compliance to reactive strategies [92], serotype carriage [99] and cellular mediated immunity [87] were incorporated. Social mixing behavior and transmission events were modeled in one unified population $[87,97]$ and/or within specific social contact clusters such as households, schools, workplaces and communities [84, 103], sometimes in combination with occasional long distance trips [92]. 


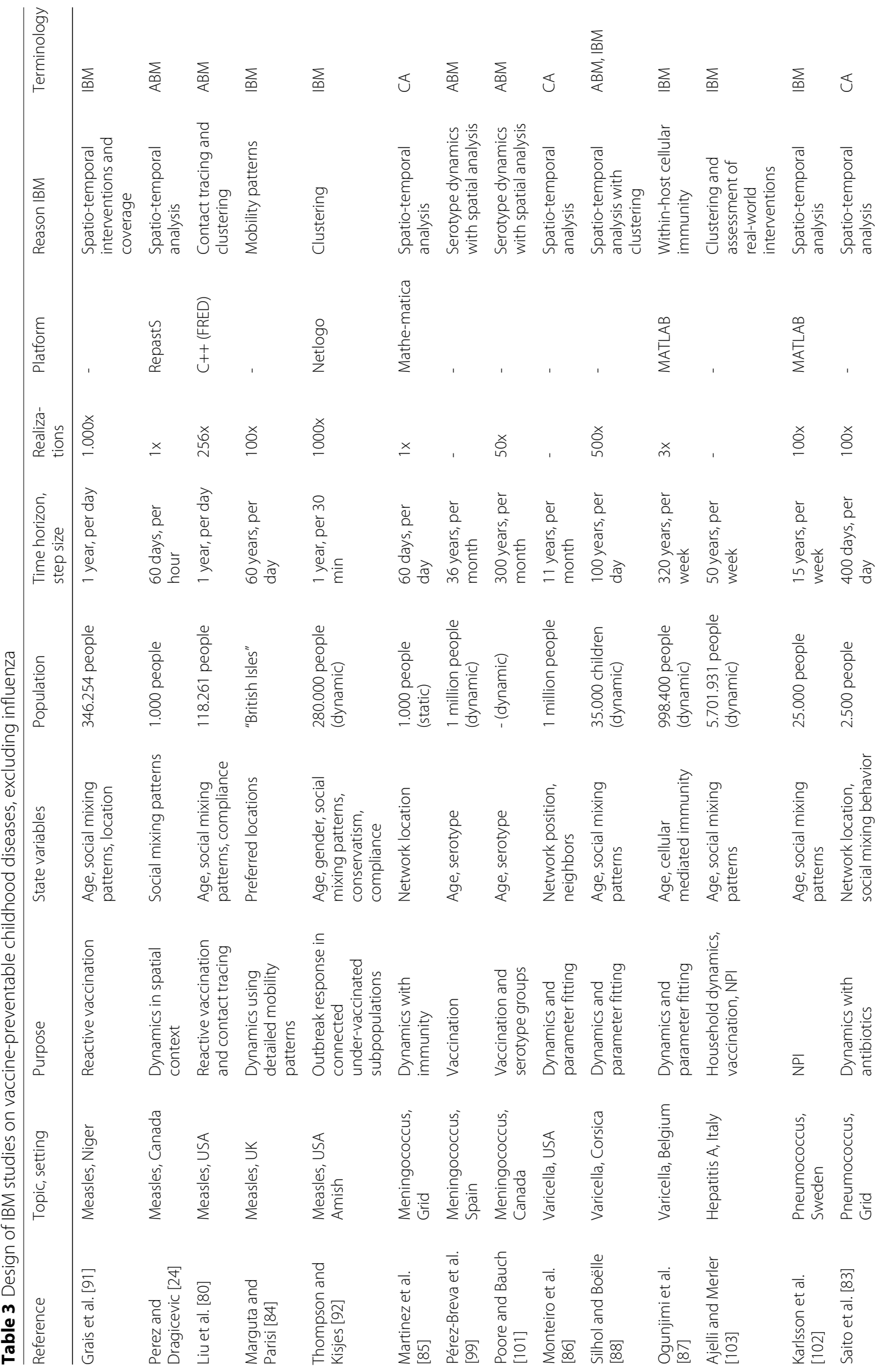




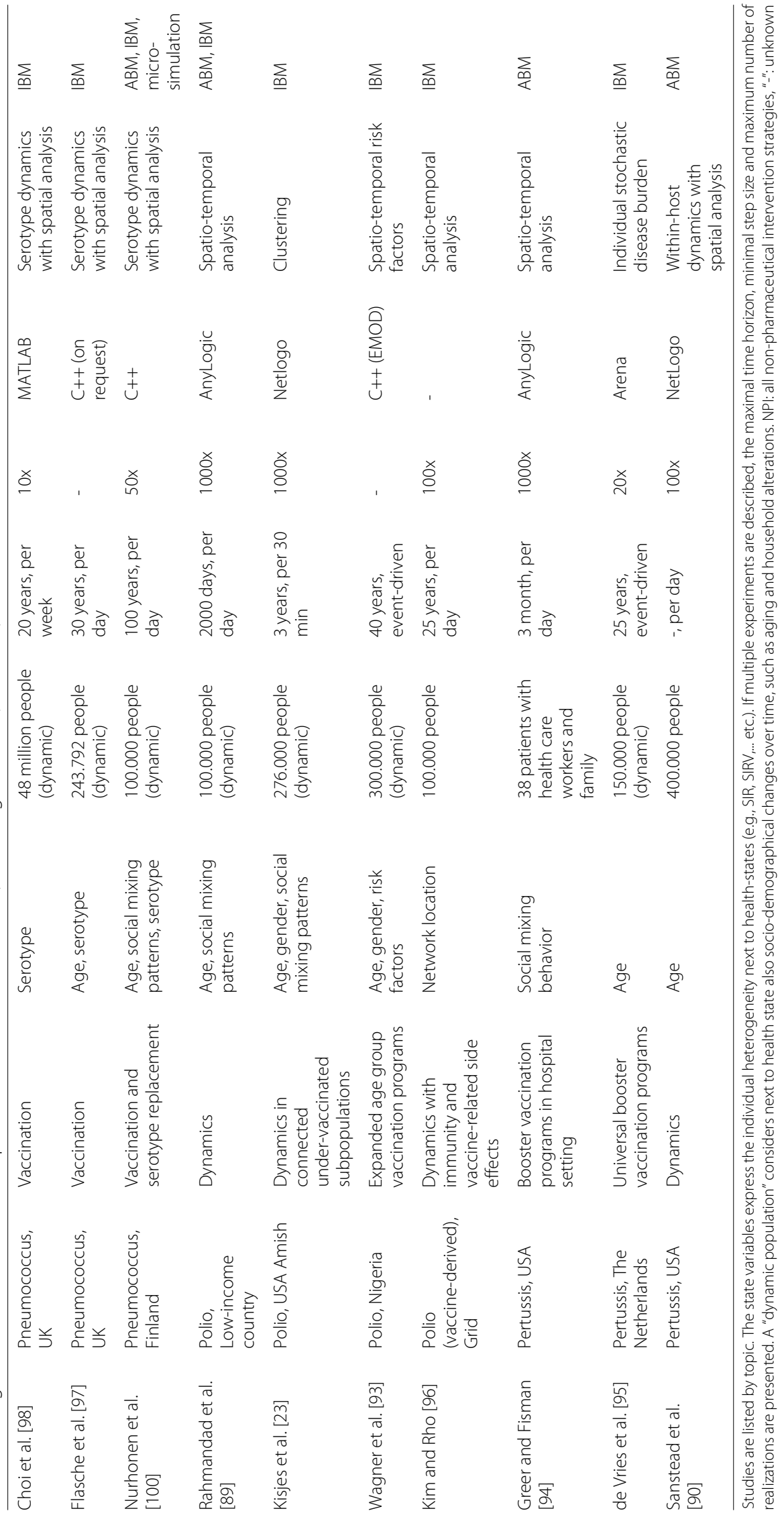




\section{Population}

The population sizes ranged from 38 infants in a hospital setting [94] up to 48 million inhabitants of England and Wales [98]. The dynamics regarding age and social mixing in the population were modeled static (i.e. constant) [85] or dynamic (with ageing, mortality, newborns, weddings) $[92,100]$. Ajelli and Merler [103] were exceptional in that they provided an explicit approach to model household dynamics over time to enable IBM simulations on long time-scales.

\section{Time horizon}

We observed a spectrum of time horizons from 60 days [24] up to 320 years [87]. The step size was mostly one day (e.g., [83, 88, 91]) but ranged from 30 minutes [23] up to one month [86]. Two event-driven models had no fixed time steps $[93,95]$.

\section{Realizations}

For stochastic IBMs, one initial condition can lead to different outcomes so multiple realizations are highly recommended. The number of realizations for each parameter set to quantify the uncertainty on the results varied in our search from 3x [87], 10x [98] and 100x [90] up to $1000 x$ [89]. For five papers, we were not able to retrieve the number of realizations $[86,93,97,99,103]$.

\section{Platform}

We distinguished a category of papers using mathematical software such as MATLAB ${ }^{\circ}$ [102] and Mathematica ${ }^{\circ}$ [85]. Others used more explicit modeling platforms for IBMs such as NetLogo [23, 90], RepastS [24] and AnyLogic [94]. One model was implemented in Arena, which is specific software for discrete-event simulations [104]. Four studies reported a model implemented in $\mathrm{C}_{+}+[80,93,97,100]$.

\section{Reason IBM}

We discerned 3 main reasons for choosing an IBM for these childhood diseases. Firstly, to model heterogeneous between-host interactions regarding social mixing behavior, age, demography, clustering, compliance to mitigation strategies and spatial distribution (e.g., [85, 88, 91]). Secondly, to model heterogeneous within-host processes in combination with between-host interactions (e.g., [87, 98, 99]). For instance, Choi et al. [98] analyzed serotype replacement and developed an IBM to track the multitude of possible vaccine states and dose combinations, which was too complex to capture in a compartmental model framework. Thirdly, to obtain stochastic individual-level information on the disease burden to inform economic analysis or other post-processing [95].

\section{Terminology}

Of the 24 articles, 12 used only "IBM" to denote their individual-level transmission model. Six papers used
"ABM" and 3 "CA". Nurhonen et al. [100] used the terms "IBM", "ABM" and micro-simulation interchangeably. Silhol and Boelle [88] and Rahmandad et al. [89] used "IBM" and "ABM".

\section{Model performance}

Only Rahmandad et al. [89] defined model requirements and performance. They reported runtimes and stated that specialized computer clusters were required to simulate very large populations. To set up the scalefree network, 30 minutes were required on an Intel Core 2 CPU 6400@2.13 GHz desktop. The runtimes to model transmission dynamics scaled with population size. A few papers mentioned that their results were obtained on a cluster $[87,89,100]$, without providing details.

\section{Other}

The amount of missing information on the platform or other technical details is noteworthy, especially when the model is not described elsewhere or open-source. Two papers provide a model name, FRED [80] and EMOD [93], and one states that the source code is available on request [97]. In some papers [83, 95, 100], model characteristics such as population size, time horizon, step size or number of realizations had to be retrieved from the "Results" or "Discussion" sections or from figure captions.

\section{Discussion}

The number of published IBMs for infectious disease transmission and the diversity of disease topics are increasing. Our systematic search identified 698 unique papers between 2006 and 2015. Most included articles were applied to unspecified close-contact infections or to influenza, though IBMs for other air-, saliva-, vectorborne and sexually transmitted infections are emerging. Methods for vector-borne diseases have been described for malaria and dengue and could guide future research. Especially, IBM applications on chikungunya and zika are expected over the next decade given the growing geographical expansion of their common vectors [49, 105]. Also screening and (non-)pharmaceutical intervention strategies have not been fully explored with IBMs for many diseases. Given the heterogeneous nature of biomedical and socioeconomic data and the accelerating health care expenditures, IBMs become progressively useful to inform policy makers, particularly in combination with efficiency and equity analyses [106, 107]. There are relatively few papers with an IBM for stochastic outbreak analysis under high vaccination coverage, for example for vaccine-preventable childhood diseases. For measles, it was shown that stochastic fluctuations around the endemic equilibrium in populations with high vaccination coverage could cause recurrent epidemics [84]. We expect future research to focus 
more on these topics with IBMs in combination with increasing global mobility, urbanization, climate change, disease elimination efforts and vaccine skepticism [2]. Customization of health care is one way to mitigate these stochastic epidemics with medical interventions tailored to the individual patient. The rising transition towards precision medicine needs to be informed with studies on the individual-level to capture spatio-temporal heterogeneity.

Modeling frameworks, such as STDSIM [71], EMOD [73] and EPISIMS [72] exist but are limited in that their application depends heavily on specific input data. Indeed, it is difficult to create or maintain generic models that incorporate many modeling options and still manage the computational burden. Nonetheless, given the high programming burden, transparent reuse of models increases confidence in their approach and generated results. Making IBM code open-source (e.g., FluTE [36] and FRED [76]) is also useful to validate model outcomes, to inspire future modeling projects [55] and to expand model exploration [108]. Consistent "branding" of the IBM, with a proper acronym, is practical to link studies and consolidate intellectual ownership of freely accessible source code.

Regarding the simulation platform, mathematical software (e.g., MATLAB ${ }^{\circ}$ ) enables many embedded features and is user-friendly but currently lacks specific modules for IBMs. Integrated platforms such as RepastS [109] and Netlogo $[25,110]$, are used by others and can be practical and straightforward but cannot fulfill all requirements of the inherent heterogeneity and computational burden of IBMs. A third option is the low-level programming language $\mathrm{C}++$, which enables high-performance code but requires high-level programming skills to efficiently manage the model logic and memory usage. Given the computational and implementation burden [84, 89], close interaction with computer sciences is required. Nonetheless, good-practice programming with version control, regression testing and benchmarking is rarely described [108, 111].

Although runtimes are inherent to model implementation and computer hardware, presenting the order of magnitude of runtimes and memory requirements could be useful for other researchers. Details on model performance and computational burden were usually lacking in our selection of full-text papers. In our total set of IBM papers, we found 2 examples on the computational burden of their IBM in $\mathrm{C}++[36,76]$. An influenza simulation with FLUTE [36] uses approximately 80 megabytes of memory per million simulated individuals. Simulating an epidemic in a population of 10 million people can take up to two hours (on a single processor on an Intel Core Duo T9400), but it may take only seconds if the virus is not highly transmissible or if there are effective interventions [36]. With
750 - 1000 megabytes of memory required per million simulated individuals, FRED's computational burden [76] is about ten times larger. Simulations for the H1N1 pandemic in a population of 1 million people takes less than two minutes on a typical dual-core laptop computer (in 2013) but the runtime will vary depending on the number of individuals infected during the epidemic and depending on which optional features are activated. Unfortunately, computational performance is a significant aspect of a simulator's usefulness. Investment in performance optimization is required to achieve the full potential of current high-performance workstations [108]. This seems most feasible using open-source software, as it allows more researchers to contribute to optimization and to leverage on the existing - and ever expanding - IBM knowledge base, thus enabling a cyclic process of innovation and optimization.

Time horizons and modeling step sizes in the full-text articles were diverse and are subject to disease characteristics and research objectives. There is no standard approach on the number of stochastic realizations, which seems model specific and requires sensitivity analysis. Models focusing on key factors of between-host dynamics in large populations with homogeneous mixing [87] will not produce much stochastic variability and require fewer realizations compared to simulations combining complex social mixing clusters, adaptive behavior, withinhost dynamics and medical backgrounds [80, 91, 92]. One of the most frequent criticisms of IBMs is that "they can be calibrated to say anything" [25]. This is partly a result of not capturing the difference between the calibration of IBMs and equation-based models. The latter have usually fewer parameters, which have to be evaluated by calibrating the full model to observed data [25]. IBMs, in contrast, are constructed bottom-up, which allows to select parameters independently based on census data, mobility patterns, serotype distribution, social contact behavior, natural history of a disease, etc. As such, a limited number of particularly uncertain parameters has to be calibrated by fitting the model to observed prevalence and/or incidence of disease states [25]. IBM calibration has been performed with genetic algorithms [86], maximum likelihood [88] or Bayesian procedures with Markov Chain Monte Carlo sampling [53]. A limitation of the IBM approach is that the basic reproduction number $\left(\mathrm{R}_{0}\right)$, corresponding to the number of secondary cases caused by a single (typical) infection in a totally susceptible population, cannot be attributed directly but has to be derived from model output. $\mathrm{R}_{0}$ has been estimated in IBM studies $[23,36,76,88,108,112]$ by the average number of secondary cases from a randomly selected individual in a fully susceptible model population based on multiple realizations. Parameterization and calibration needs to be documented well. Model presentation should preferably 
be accompanied by an assessment of the goodness of fit to observed data [4]. Another convincing way to show that your ABM has been calibrated without bias and produces useful general results is to analyze it thoroughly after calibration [25]. Ideally, each model should be analyzed systematically to understand the impact of model assumptions and parameters on the results [55]. Parameter values can be drawn from a pre-computed design (e.g., Latin Hypercube) or at random from a distribution. Emulation techniques are promising to capture complex simulators' behavior in order to improve engaged and perhaps more rapid policy making [55-58]. Given the lack of standards, it is crucial to fully describe the methods and experimental design in the context of the model [4]. Unfortunately, we were not able to recapture all model characteristics and study designs from our full-text subset. This stresses the need for the ODD protocol with shorthand conventions and a syntax that modelers can understand intuitively such that the methodology can be converted directly into an executable simulator [28].

The terminology to describe individual-level models and infectious diseases was inconsistent and curtail efficient knowledge transfer. For example, a systematic review in 2015 on IBMs for non-communicable diseases [33] searched only with the terms "agent-based" and "individual-based" to dramatically reduce the number of false positive hits. To assist future research, it is crucial to use the same semantics for IBM studies across disciplines. The introduction of the Medical Subject Headings (MeSH) controlled vocabulary [113] is a huge step forward but is limited to PubMed and does not (yet) contain fixed terms for simulation models at the individual-level. With this review, we seek to provide keywords to the IBM community and a definition for individual-based modeling as "computer simulation for the creation, disappearance and movement of a finite collection of interacting individuals or agents with unique attributes regarding spatial location, physiological traits and/or social behavior" [8-11, 25, 33]. The overall term IBM refers to the individual-level approach based on a fundamental philosophy of methodological individualism, which advocates a focus on the uniqueness of individuals and their interactions. Further subcategories can be used according to whether locations are static (as in CAs) or individuals act autonomously (as in ABMs). The standard incorporation of the overarching term "individual-based model" in the abstract or keywords would greatly improve current and future systematic searches in large electronic databases.

One could argue that our recommendations are constrained since they are based on title, abstract and keyword screening. For example, a frequently cited article on pandemic influenza by Ferguson et al. [114] was not retrieved by our search since it has none of the IBM terms in its title, abstract or keywords. The model is described as a "large-scale epidemic simulation" in the abstract although the first sentence of the introduction reads "We parameterize an individual-based model of pandemic influenza transmission...". This example could be seen as a confirmation of the inconsistencies and limitations of current article archiving practice. A similar remark can be made for our disease related search terms but we believe we used the most relevant keywords and can only recommend future research to include also general disease-related terminology. If our selection was identifiable by searching on "infectious AND disease AND transmission", this would be a substantial improvement, in contrast to the current $40 \%$ of our selection. The restriction to only include papers published between 2006 and 2015 might be considered a minor limitation by the time the current paper is published. The final fully included year was chosen mainly for practical reasons, at the time of completing this labour-intensive review in early 2016. We are convinced that a review over a decade (an intuitively appealing period for review) is highly informative to understand the evolution of this field and to adequately guide future research. We had no intention to present a complete review of all the IBM papers we systematically identified, but we provide all included references in a searchable database enabling others to conduct more specific literature reviews. Clearly, our database can be updated using the discussed insights on search methodology and keywords.

\section{Conclusion}

We systematically reviewed a decade of recent literature on infectious disease transmission IBMs and propose a common terminology to facilitate knowledge transfer within and across disciplines. IBMs have already been useful to explore heterogeneous between-host interactions both with and without unique within-host (dynamic) processes. The number of IBMs to study transmission and control of HIV, HPV, malaria and tuberculosis is increasing. The combination of targeted screening and vaccination strategies with economic evaluations is promising for the near future. Emerging diseases are the dominant applications in infectious disease IBMs. Notwithstanding, similar models are required for endemic diseases, such as vaccine-preventable childhood diseases, to capture stochastic and heterogeneous characteristics, which are especially relevant in the final stages of elimination. We provide 698 unique references published between 20062015 with study characteristics to inform the research community across topics and terminology. We recommend cooperation in open-source projects and adhering to the ODD protocol, which enables modelers to describe their IBM using a common syntax. Common model-names enhance the research community's ability to grasp common features between models, and discover 
opportunities for further model improvements. Transfer of expertise on IBMs is required to capitalize future research opportunities, which is facilitated through the increasing availability of individual-level data and the rising interest for precision medicine. In this respect, the combination of screening and targeted vaccination strategies with economic evaluations seems an interesting future prospect.

\section{Additional files}

Additional file 1: PRISMA flow diagram. Adapted PRISMA flow diagram of the systematic review process. (PDF $52 \mathrm{~kb}$ )

Additional file 2: Reference list. List of the included studies using an individual-based model to study infectious disease transmission. References are sorted by topic and disease and listed together with their terminology, modeling purpose, (non)inclusion of an economic analysis, intervention strategy and methodology classification. (CSV 183 kb)

Additional file 3: The relative number of IBM studies over time. Supporting information on additional literature searches to estimate the relative number of IBM studies for infectious disease transmission over time compared to modeling studies for infectious disease transmission in general. (PDF 93 kb)

\section{Abbreviations}

ABM: Agent-based model; CA: Cellular automata; HIV: Human immunodeficiency virus; HPV: Human papillomavirus; IBM: Individual-based model; ODD: Overview, Design concepts, and Details (protocol)

\section{Acknowledgements}

The authors acknowledge support of the Antwerp Study Centre for Infectious Diseases (ASCID) at the University of Antwerp.

\section{Funding}

LW and FV are supported by the Research Foundation Flanders (FWO, G043815N). JB is supported by a postdoctoral grant from the Research Foundation Flanders (FWO). NH acknowledges support from the University of Antwerp scientific chair in Evidence-Based Vaccinology, financed in 2009-2017 by a gift from Pfizer and GSK. The funders had no role in study design, data collection and analysis, decision to publish, or preparation of the manuscript.

\section{Availability of data and materials}

All data generated or analyzed during this study are included in this published article and its supplementary information files.

\section{Authors' contributions}

PB initiated the study. LW FV JB NH and PB conceived and designed the inclusion criteria. LW and FV performed the screening. LW FV JB NH and PB wrote the paper. All authors read and approved the final manuscript.

\section{Ethics approval and consent to participate}

"Not applicable".

\section{Consent for publication}

"Not applicable".

\section{Competing interests}

The authors declare that they have no competing interests.

\section{Publisher's Note}

Springer Nature remains neutral with regard to jurisdictional claims in published maps and institutional affiliations.

\section{Author details}

${ }^{1}$ Centre for Health Economics Research \& Modeling Infectious Diseases, Vaccine and Infectious Disease Institute, University of Antwerp, Antwerp,
Belgium. ${ }^{2}$ Interuniversity Institute for Biostatistics and statistical Bioinformatics, UHasselt, Hasselt, Belgium. ${ }^{3}$ School of Public Health and Community Medicine, The University of New South Wales, Sydney, Australia.

Received: 25 November 2016 Accepted: 22 August 2017

Published online: 11 September 2017

\section{References}

1. Lessler J, Edmunds WJ, Halloran ME, Hollingsworth TD, Lloyd AL. Seven challenges for model-driven data collection in experimental and observational studies. Epidemics. 2014;10:3-7.

2. Holzmann $\mathrm{H}$, Hengel $\mathrm{H}$, Tenbusch $\mathrm{M}$, Doerr $\mathrm{H}$. Eradication of measles: remaining challenges. Med Microbiol Immun. 2016;205:201-208.

3. Megiddo I, Colson AR, Nandi A, Chatterjee S, Prinja S, Khera A Laxminarayan R. Analysis of the Universal Immunization Programme and introduction of a rotavirus vaccine in India with IndiaSim. Vaccine. 2014;32:151-61.

4. Garnett G, Cousens S, Hallet $T$, Steketee R, Walker N. Mathematical models in the evaluation of health programmes. Lancet. 2011;378(9790) 515-25.

5. Mossong J, Hens N, Jit M, Beutels P, Auranen K, Mikolajczyk R, Massari M, Salmaso S, Tomba GS, Wallinga J, Heijne J, Sadkowska-Todys M, Rosinska M, Edmunds WJ. Social contacts and mixing patterns relevant to the spread of infectious diseases. PLoS Med. 2008;5(3):74.

6. Salathé M, Jones JH. Dynamics and control of diseases in networks with community structure. PLoS Comput Biol. 2010;6(4):1000736.

7. Willem L, Van Kerckhove K, Chao DL, Hens N, Beutels P. A nice day for an infection? Weather conditions and social contact patterns relevant to influenza transmission. PLOS ONE. 2012;7(11):48695.

8. DeAngelis DL, Grimm V. Individual-based models in ecology after four decades. F1000Prime Rep. 2014;6:39.

9. Jit M, Brisson M. Modelling the epidemiology of infectious diseases for decision analysis. PharmacoEconomics. 2011;29(5):371-86.

10. van Kleef E, Robotham JV, Jit M, Deeny SR, Edmunds WJ. Modelling the transmission of healthcare associated infections: a systematic review. BMC Infect Dis. 2013;13(1):294

11. Roughgarden J. Individual Based Models in Ecology: An Evaluation, or How Not to Ruin a Good Thing. Philos Sci Ass Bienn Meet. 2012;9434.

12. Funk S, Salathé M, Jansen VA. Modelling the influence of human behaviour on the spread of infectious diseases: a review. J R Soc Interface. 2010;7(50):1247-56.

13. Verelst $F$, Willem $L$, Beutels P. Behavioural change models for infectious disease transmission: a systematic review (2010-2015). J R Soc Interface. 2016;13(125):20160820.

14. Funk S, Camacho A, Kucharski AJ, Eggo RM, Edmunds WJ. Real-time forecasting of infectious disease dynamics with a stochastic semi-mechanistic model. Epidemics. 2016. doi:10.1016/j.epidem.2016.11.003.

15. Colizza V, Barrat A, Barthélemy M, Vespignani A. The modeling of global epidemics: Stochastic dynamics and predictability. Bull Math Biol. 2006;68(8):1893-921.

16. Balcan D, Gonçalves B, Hu H, Ramasco JJ, Colizza V, Vespignani A Modeling the spatial spread of infectious diseases: The GLobal Epidemic and Mobility computational model. J Comput Sci. 2010;1(3):132-45.

17. Bajardi P, Poletto C, Ramasco JJ, Tizzoni M, Colizza V, Vespignani A. Human mobility networks, travel restrictions, and the global spread of 2009 H1N1 pandemic. PLoS ONE. 2011;6(1):16591.

18. Tizzoni M, Bajardi P, Poletto C, Ramasco JJ, Balcan D, Gonçalves B, Perra N, Colizza V, Vespignani A. Real-time numerical forecast of global epidemic spreading: Case study of 2009 A/H1N1pdm. BMC Med. 2012;10(1):165.

19. Poletto C, Gomes MF, y Piontti AP, Rossi L, Bioglio L, Chao DL, Longini IM, Halloran ME, Colizza V, Vespignani A. Assessing the impact of travel restrictions on international spread of the 2014 West African Ebola epidemic. Euro Surveill. 2014;19(42):20936.

20. Omer SB, Salmon DA, Orenstein WA, Dehart MP, Halsey N. Vaccine refusal, mandatory immunization, and the risks of vaccine-preventable diseases. N Engl J of Med. 2009;360(19):1981-8.

21. Byington $\mathrm{CL}$, Clayton EW, Edwards KM. Childhood vaccine exemptions: A broader perspective is required. Pediatr. 2016;137(4):2016.

22. Hanley SJ, Yoshioka E, Ito Y, Kishi R. Hpv vaccination crisis in japan. Lancet. 2015;385(9987):2571. 
23. Kisjes KH, Tebbens RJD, Wallace GS, Pallansch MA, Cochi SL, Wassilak SGF, Thompson KM. Individual-Based Modeling of Potential Poliovirus Transmission in Connected Religious Communities in North America With Low Uptake of Vaccination. J Infect Dis. 2014;210:424-33.

24. Perez $\mathrm{L}$, Dragicevic $S$. An agent-based approach for modeling dynamics of contagious disease spread. Int J Health Geogr. 2009;8:50.

25. Railsback SF, Grimm V. Agent-based and individual-based modeling: a practical introduction. New Jersey: Princeton University Press; 2011.

26. Vynnycky E, White R. An introduction to infectious disease modelling. New York: OUP Oxford; 2010.

27. Grimm V. Ten years of individual-based modelling in ecology: what have we learned and what could we learn in the future? Ecol Modell. 1999;115(2):129-48.

28. Grimm V, Berger U, Bastiansen F, Eliassen S, Ginot V, Giske J, Goss-Custard J, Grand T, Heinz SK, Huse G, et al. A standard protocol for describing individual-based and agent-based models. Ecol modell. 2006;198(1):115-26.

29. Grimm V, Berger U, DeAngelis DL, Polhill JG, Giske J, Railsback SF. The ODD protocol: a review and first update. Ecol Modell. 2010;221(23): 2760-8.

30. Moher D, Liberati A, Tetzlaff J, Altman DG. Preferred reporting items for systematic reviews and meta-analyses: the PRISMA statement. Ann Intern Med. 2009;151(4):264-9.

31. Higgins JP, Green S, et al. Cochrane handbook for systematic reviews of interventions: Wiley Online Library; 2008, p. 5.

32. World Health Organization. Infectious Diseases. 2015. http://www.who. int/topics/infectious_diseases/en. Accessed on 1 Dec 2015.

33. Nianogo RA, Arah OA. Agent-based modeling of noncommunicable diseases: a systematic review. Am J Public Health. 2015;105(3):20-31.

34. Centers for Disease Control and Prevention: Recommended immunization schedule for children between birth and 15 months, united states. 2017. http://www.cdc.gov/vaccines/schedules/hcp/imz/ child-adolescent.html. Accessed on 1 June 2017.

35. Nsoesie E, Mararthe M, Brownstein J. Forecasting peaks of seasonal influenza epidemics. PLoS Curr. 2013;5.

36. Chao DL, Halloran ME, Obenchain VJ, Longini IM. FluTE, a publicly available stochastic influenza epidemic simulation model. PLoS Comput Biol. 2010;6(1):1000656

37. Halder N, Kelso JK, Milne GJ. Developing guidelines for school closure interventions to be used during a future influenza pandemic. BMC Infect Dis. 2010;10:221.

38. Lee BY, Brown ST, Korch GW, Cooley PC, Zimmerman RK, Wheaton WD, Zimmer SM, Grefenstette JJ, Bailey RR, Assi TM, Burke DS. A computer simulation of vaccine prioritization, allocation, and rationing during the 2009 H1N1 influenza pandemic. Vaccine. 2010;28(31):4875-9.

39. Merler S, Ajelli M, Fumanelli L, Gomes MFC, Piontti APY, Rossi L, Chao DL, Longini IM, Halloran ME, Vespignani A. Spatiotemporal spread of the 2014 outbreak of Ebola virus disease in Liberia and the effectiveness of non-pharmaceutical interventions: a computational modelling analysis. Lancet Infect Dis. 2015;15(2):204-11.

40. Siettos C, Anastassopoulou C, Russo L, Grigoras C, Mylonakis E. Modeling the 2014 Ebola Virus Epidemic - Agent-Based Simulations, Temporal Analysis and Future Predictions for Liberia and Sierra Leone. PLoS Curr. 2015;7.

41. Burke DS, Epstein JM, Cummings DA, Parker Jl, Cline KC, Singa RM, Chakravarty S. Individual-based computational modeling of smallpox epidemic control strategies. Acad Emerg Med. 2006;13(11):1142-9.

42. Chen LC, Carley KM, Fridsma D, Kaminsky B, Yahja A. Model alignment of anthrax attack simulations. Decis Support Syst. 2006;41(3):654-68.

43. Williams ADC, Hall IM, Rubin GJ, Amlot R, Leach S. An individual-based simulation of pneumonic plague transmission following an outbreak and the significance of intervention compliance. Epidemics. 2011;3(2): 95-102.

44. Milne GJ, Baskaran P, Halder N, Karl S, Kelso J. Pandemic influenza in Papua New Guinea: a modelling study comparison with pandemic spread in a developed country. BMJ Open. 2013;3(3):e002518.

45. Laskowski M, Xiao Y, Charland N, Moghadas SM. Strategies for Early Vaccination During Novel Influenza Outbreaks. Sci Rep. 2015;5:18062.

46. Ross A, Penny M, Maire N, Studer A, Carneiro I, Schellenberg D, Greenwood B, Tanner M, Smith T. Modelling the Epidemiological Impact of Intermittent Preventive Treatment against Malaria in Infants. PLOS ONE. 2008;3(7):e2661.
47. Maire N, Shillcutt SD, Walker DG, Tediosi F, Smith TA. Cost-Effectiveness of the Introduction of a Pre-Erythrocytic Malaria Vaccine into the Expanded Program on Immunization in Sub-Saharan Africa: Analysis of Uncertainties Using a Stochastic Individual-Based Simulation Model of Plasmodium falciparum Malaria. Value Health. 2011;14(8):1028-38.

48. Penny MA, Galactionova K, Tarantino M, Tanner M, Smith TA. The public health impact of malaria vaccine RTS, S in malaria endemic Africa: country-specific predictions using 18 month follow-up Phase III data and simulation models. BMC Med. 2015;13:170.

49. Karl S, Halder N, Kelso JK, Ritchie SA, Milne GJ. A spatial simulation model for dengue virus infection in urban areas. BMC Infect Dis. 2014; 14:447.

50. Poletti P, Merler S, Ajelli M, Manfredi P, Munywoki PK, Nokes DJ, Melegaro A. Evaluating vaccination strategies for reducing infant respiratory syncytial virus infection in low-income settings. BMC Med. 2015;13(1):1.

51. Li M, Ma J, van den Driessche P. Model for disease dynamics of a waterborne pathogen on a random network. J Math Biol. 2015;71(4): $961-77$.

52. Prieto D, Das TK. An operational epidemiological model for calibrating agent-based simulations of pandemic influenza outbreaks. Health Care Manag Sci. 2014;19(1):1-19.

53. Cameron E, Battle KE, Bhatt S, Weiss DJ, Bisanzio D, Mappin B, Dalrymple U, Hay SI, Smith DL, Griffin JT, Wenger EA, Eckhoff PA, Smith TA, Penny MA, Gething PW. Defining the relationship between infection prevalence and clinical incidence of Plasmodium falciparum malaria. Nat Commun. 2015;6:8170.

54. Osgood N. Lightening the performance burden of individual-based models through dimensional analysis and scale modeling. Syst Dynam Rev. 2009;25(2):101-34.

55. Willem L, Stijven S, Vladislavleva E, Broeckhove J, Beutels P, Hens N. Active learning to understand infectious disease models and improve policy making. PLoS Comput Biol. 2014;10(4):1003563.

56. Keeling MJ, Ross JV. On methods for studying stochastic disease dynamics. J R Soc Interface. 2008;5(19):171-81.

57. Buckeridge $\mathrm{DL}$, Jauvin $\mathrm{C}$, Okhmatovskaia A, Verma AD. Simulation Analysis Platform (SnAP): a tool for evaluation of public health surveillance and disease control strategies. Proc AMIA Annu Symp. 2011;2011:161-70.

58. Kasaie P, Mathema B, Kelton WD, Azman AS, Pennington J, Dowdy DW. A Novel Tool Improves Existing Estimates of Recent Tuberculosis Transmission in Settings of Sparse Data Collection. PLoS ONE. 2015;10(12):0144137.

59. Leclerc PM, Matthews AP, Garenne ML. Fitting the HIV Epidemic in Zambia: A Two-Sex Micro-Simulation Model. PLoS ONE. 2009;4(5):e5439.

60. Botari T, Alves SG, Leonel ED. Explaining the high number of infected people by dengue in Rio de Janeiro in 2008 using a susceptible-infective-recovered model. Phys Rev E. 2011;83(3):037101.

61. Guzzetta G, Ajelli M, Yang ZH, Merler S, Furlanello C, Kirschner D. Modeling socio-demography to capture tuberculosis transmission dynamics in a low burden setting. J Theor Biol. 2011;289:197-205.

62. Ajelli M, Gonçalves B, Balcan D, Colizza V, Hu H, Ramasco JJ, Merler S, Vespignani A. Comparing large-scale computational approaches to epidemic modeling: agent-based versus structured metapopulation models. BMC Infec Dis. 2010;10(1):190.

63. Chao DL, Halstead SB, Halloran ME, Longini Jr IM. Controlling dengue with vaccines in Thailand. PLoS Negl Trop Dis. 2012;6(10):1876.

64. Lawniczak AT, Fuks H, Di Stefano B. Individually based SIR models, their motivation, and their spatio-temporal dynamics. DCDIS Series A Math Anal. 2006;13:437-55.

65. Nunes A, da Gama MT, Gomes M. Localized contacts between hosts reduce pathogen diversity. J Theor Biol. 2006;241 (3):477-87.

66. Germann TC, Kadau K, Longini Jr IM, Macken CA. Mitigation strategies for pandemic influenza in the United States. Proc Natl Acad Sci. 2006;103(15):5935-40.

67. Reppas Al, Spiliotis KG, Siettos Cl. Epidemionics: from the host-host interactions to the systematic analysis of the emergent macroscopic dynamics of epidemic networks. Virulence. 2010;1 (4):338-49.

68. Andradóttir S, Chiu W, Goldsman D, Lee LM, Tsui KL, Sander B, Fisman DN, Nizam A. Reactive strategies for containing developing outbreaks of pandemic influenza. BMC Public Health. 2011;11(1):1. 
69. Halloran ME, Ferguson NM, Eubank S, Longini IM, Cummings DAT, Lewis B, Xu SF, Fraser C, Vullikanti A, Germann TC, Wagener D, Beckman R, Kadau K, Barrett C, Macken CA, Burke DS, Cooley P. Modeling targeted layered containment of an influenza pandemic in the United States. Proc Natl Acad Sci USA. 2008;105(12):4639-44.

70. Thavara U, Tawatsin A, Nagao Y. Simulations to compare efficacies of tetravalent dengue vaccines and mosquito vector control. Epidem Infect. 2014;142(6):1245-58.

71. Orroth KK, Freeman EE, Bakker R, Buvé A, Glynn JR, Boily MC, White RG, Habbema JDF, Hayes RJ. Understanding the differences between contrasting HIV epidemics in east and west Africa: results from a simulation model of the Four Cities Study. Sex Transm Inf. 2007; 83(suppl 1):5-16.

72. Stroud P, Del Valle S, Sydoriak S, Riese J, Mniszewski S. Spatial dynamics of pandemic influenza in a massive artificial society. J Artif Soc Soc Sim. 2007;10(4):9.

73. Bershteyn A, Klein DJ, Eckhoff PA. Age-dependent partnering and the HIV transmission chain: a microsimulation analysis. J R Soc Interf. 2013;10(88):20130613.

74. Coffeng LE, StolkWA, Zoure HG, Veerman JL, Agblewonu KB, Murdoch ME, Noma M, Fobi G, Richardus JH, Bundy DA, et al. African programme for onchocerciasis control 1995-2015: model-estimated health impact and cost. PLoS Negl Trop Dis. 2013;7(1):2032.

75. Brisson M, Laprise JF, Drolet M, Van de Velde N, Franco EL, Kliewer EV, Ogilvie G, Deeks SL, Boily MC. Comparative cost-effectiveness of the quadrivalent and bivalent human papillomavirus vaccines: a transmission-dynamic modeling study. Vaccine. 2013;31(37):3863-871.

76. Grefenstette JJ, Brown ST, Rosenfeld R, DePasse J, Stone NT, Cooley PC, Wheaton WD, Fyshe A, Galloway DD, Sriram A, Guclu H, Abraham T, Burke DS. FRED (A Framework for Reconstructing Epidemic Dynamics): An open-source software system for modeling infectious diseases and control strategies using census-based populations. BMC Public Health. 2013;13(1):940.

77. Stuckey EM, Stevenson JC, Cooke MK, Owaga C, Marube E, Oando G, Hardy D, Drakeley C, Smith TA, Cox J, et al. Simulation of malaria epidemiology and control in the highlands of western Kenya. Malar J. 2012;11(357):10-1186.

78. Matthijsse SM, van Rosmalen J, Hontelez JA, Bakker R, de Kok IM, van Ballegooijen M, de Vlas SJ. The role of acquired immunity in the spread of human papillomavirus (HPV): Explorations with a microsimulation model. PloS ONE. 2015;10(2):0116618.

79. White R, Freeman E, Orroth K, Bakker R, Weiss H, O'Farrell N, Buvé A, Hayes R, Glynn J. Population-level effect of HSV-2 therapy on the incidence of HIV in sub-Saharan Africa. Sex Transm Infect. 2008; 84(Suppl 2):12-18.

80. Liu FC, Enanoria WTA, Zipprich J, Blumberg S, Harriman K, Ackley SF, Wheaton WD, Allpress JL, Porco TC. The role of vaccination coverage, individual behaviors, and the public health response in the control of measles epidemics: an agent-based simulation for California. BMC Public Health. 2015;15:447

81. Klein DJ, Eckhoff PA, Bershteyn A. Targeting HIV services to male migrant workers in southern Africa would not reverse generalized HIV epidemics in their home communities: a mathematical modeling analysis. Int Health. 2015;7(2):107-13.

82. Gerardin J, Ouédraogo AL, McCarthy KA, Eckhoff PA, Wenger EA. Characterization of the infectious reservoir of malaria with an agent-based model calibrated to age-stratified parasite densities and infectiousness. Malar J. 2015;14(1):231.

83. Saito Y, da Silva MA, Alves D. A Probabilistic Cellular Automata to Studying the Spreading of Pneumonia in a Population. Biomat 2009. 2010;354-65.

84. Marguta R, Parisi A. Impact of human mobility on the periodicities and mechanisms underlying measles dynamics. J R Soc Interface. 2015;12(104):20141317.

85. Martinez MJF, Merino EG, Sanchez EG, Sanchez JEG, del Rey AM, Sanchez GR. A mathematical model to study the meningococcal meningitis. 2013 Inter Conf Comput Sci. 2013;18:2492-495.

86. Monteiro LHA, Oliveira DN, Chaui-Berlinck JG. The Effect of Spatial Scale on Predicting Time Series: A Study on Epidemiological System Identification. Math Probl Eng. 2009;2009:10. Article ID 137854
87. Ogunjimi B, Willem L, Beutels P, Hens N. Integrating between-host transmission and within-host immunity to analyze the impact of varicella vaccination on zoster. Elife. 2015;4:07116.

88. Silhol R, Boelle PY. Modelling the Effects of Population Structure on Childhood Disease: The Case of Varicella. PLoS Comput Biol. 2011;7(7): e1002105.

89. Rahmandad H, Hu K, Tebbens RJD, Thompson KM. Development of an individual-based model for polioviruses: implications of the selection of network type and outcome metrics. Epidem Infect. 2011;139(6):836-48.

90. Sanstead E, Kenyon C, Rowley S, Enns E, Miller C, Ehresmann K, Kulasingam S. Understanding Trends in Pertussis Incidence: An Agent-Based Model Approach. Am J Public Health. 2015;105(9):42-7.

91. Grais RF, Conlan AJK, Ferrari MJ, Djibo A, Le Menach A, Bjornstad ON, Grenfell BT. Time is of the essence: exploring a measles outbreak response vaccination in Niamey, Niger. J R Soc Interface. 2008;5(18): 67-74.

92. Thompson KM, Kisjes KH. Modeling measles transmission in the north american amish and options for outbreak response. Risk Anal. 2015;36(7):1404-17.

93. Wagner BG, Behrend MR, Klein DJ, Upfill-Brown AM, Eckhoff PA, Hu H. Quantifying the impact of expanded age group campaigns for polio eradication. PLOS ONE. 2014:9(12):113538.

94. Greer AL, Fisman DN. Keeping vulnerable children safe from pertussis: preventing nosocomial pertussis transmission in the neonatal intensive care unit. Infect Con Hosp Epidem. 2009:30(11):1084-9.

95. de Vries R, Kretzschmar M, Schellekens JFP, Versteegh FGA, Westra TA, Roord JJ, Postma MJ. Cost-effectiveness of adolescent pertussis vaccination for the Netherlands: using an individual-based dynamic model. PLOS ONE. 2010;5(10):e13392.

96. Kim JH, Rho SH. Transmission dynamics of oral polio vaccine viruses and vaccine-derived polioviruses on networks. J Theor Biol. 2015;364:266-74.

97. Flasche S, Edmunds WJ, Miller E, Goldblatt D, Robertson C, Choi YH. The impact of specific and non-specific immunity on the ecology of Streptococcus pneumoniae and the implications for vaccination. Proc Biol Sci. 2013;280(1771):20131939.

98. Choi YH, Jit M, Flasche S, Gay N, Miller E. Mathematical modelling long-term effects of replacing prevnar7 with prevnar13 on invasive pneumococcal diseases in England and Wales. PLoS ONE. 2012;7(7): e39927.

99. Perez-Breva L, Villanueva RJ, Villanueva-Oller J, Acedo L, Santonja F, Morano JA, Abad R, Vazquez JA, Diez-Domingo J. Optimizing strategies for meningococcal C disease vaccination in Valencia (Spain). BMC Infect Dis. 2014;14:280.

100. Nurhonen M, Cheng AC, Auranen K. Pneumococcal transmission and disease in silico: a microsimulation model of the indirect effects of vaccination. PLoS ONE. 2013;8(2):56079.

101. Poore KD, Bauch CT. The impact of aggregating serogroups in dynamic models of Neisseria meningitidis transmission. BMC Infect Dis. 2015;15: 300.

102. Karlsson D, Jansson A, Normark BH, Nilsson P. An individual-based network model to evaluate interventions for controlling pneumococcal transmission. BMC Infect Dis. 2008:8:83.

103. Ajelli M, Merler S. An individual-based model of hepatitis A transmission. J Theor Biol. 2009:259(3):478-88.

104. Kelton WD. Simulation with arena. New York: McGraw-Hill; 2002.

105. Dommar CJ, Lowe R, Robinson M, Rodó X. An agent-based model driven by tropical rainfall to understand the spatio-temporal heterogeneity of a chikungunya outbreak. Acta Tropica. 2014;129:61-73.

106. Ozawa S, Clark S, Portnoy A, Grewal S, Brenzel L, Walker DG. Return On Investment From Childhood Immunization In Low-And Middle-Income Countries, 2011-20. Health Aff. 2016;35(2):199-207.

107. Luyten J, Beutels P. The social value of vaccination programs: beyond cost-effectiveness. Health Aff. 2016;35(2):212-8.

108. Willem L, Stijven S, Tijskens E, Beutels P, Hens N, Broeckhove J. Optimizing agent-based transmission models for infectious diseases,. BMC Bioinforma. 2015;16:183.

109. North MJ, Collier NT, Ozik J, Tatara ER, Macal CM, Bragen M, Sydelko P. Complex adaptive systems modeling with repast simphony. Compl dapt Syst Model. 2013;1 (1):3.

110. Wilensky U. Center for Connected Learning and Computer-Based Modeling, Northwestern University, Evanston, IL: Netlogo; 1999. http:// ccl.northwestern.edu/netlogo/. 
111. Grimm V, Augusiak J, Focks A, Frank BM, Gabsi F, Johnston AS, Liu C, Martin BT, Meli M, Radchuk V, et al. Towards better modelling and decision support: documenting model development, testing, and analysis using TRACE. Ecol Modell. 2014;280:129-39.

112. Kuylen E, Stijven S, Broeckhove J, Willem L. Social contact patterns in an individual-based simulator for the transmission of infectious diseases (stride). Procedia Comput Sci. 2017;108:2438-442.

113. Rogers F. Medical subject headings,. Bull Med Libr Assoc. 1963;51:114.

114. Ferguson NM, Cummings DAT, Fraser C, Cajka JC, Cooley PC, Burke DS. Strategies for mitigating an influenza pandemic. Nature. 2006;442(7101): $448-52$.

\section{Submit your next manuscript to BioMed Central} and we will help you at every step:

- We accept pre-submission inquiries

- Our selector tool helps you to find the most relevant journal

- We provide round the clock customer support

- Convenient online submission

- Thorough peer review

- Inclusion in PubMed and all major indexing services

- Maximum visibility for your research

Submit your manuscript at www.biomedcentral.com/submit 